\title{
Obesity, the Obesity Epidemic, and Metabolic Dysfunction: The Conundrum Presented by the Disconnect between Evolution and Modern Societies
}

\author{
David A. Hart ${ }^{1,2,3,4}$
}

${ }^{1}$ Bone \& Joint Health Strategic Clinical Network, Alberta Health Services, Edmonton, Canada; ${ }^{2}$ Department of Surgery and Faculty of Kinesiology, University of Calgary, Calgary, Canada; ${ }^{3}$ McCaig Institute for Bone \& Joint Health, University of Calgary, Calgary, Canada; ${ }^{4}$ Centre for Hip Health \& Mobility, University of British Columbia, Vancouver, Canada

Correspondence to: David A. Hart, hartd@ucalgary.ca Keywords: Obesity, Obesity Epidemic, Metabolic Disturbances, Evolution, Characteristics of Obesity, Causes of Obesity

Received: April 7, $2021 \quad$ Accepted: May 11, $2021 \quad$ Published: May 14, 2021

Copyright $\odot 2021$ by author(s) and Scientific Research Publishing Inc.

This work is licensed under the Creative Commons Attribution International License (CC BY 4.0).

http://creativecommons.org/licenses/by/4.0/

\section{(c) (1) Open Access}

\section{ABSTRACT}

Currently, there is an obesity epidemic in the developed world, with both adults and children being affected. The consequences of this epidemic on health and health outcomes have impact at multiple levels, and it is increasing. The basis for this epidemic, which appears to have emerged with significance $\sim 40$ - 50 years ago, is unknown but is believed by many to have much of its basis in poor diets and inactivity/sedentary behaviour. Analysis of the human genome has revealed $>100$ loci which exhibit risk for development of obesity. Why there are so many loci, and how they benefited humans evolutionarily are unknown. In spite of these limitations, there are urgent needs for effective short-term interventions to assist those with obesity, as well as longer-term needs to effectively prevent development of obesity. For the former, personalized exercise programs, use of prebiotics, optimal nutrition and surgical interventions can be effective for some individuals but more interventions that address cause are also needed. For longer term solutions more detailed genetic and epigenetic understanding of risk will be required. An attractive speculation is that the genomic risk factors for obesity ( $>100$ identified) have been retained evolutionarily to address acute metabolic needs and current conditions have converted such risks to a chronic state, making reversal more difficult and with more consequences, including possible epigenetic modifications of risk genes. Other contributing factors to chronic obesity could also relate to chemical disruptors in the environment over the past $50+$ years which may impact metabolic regulation via the established risk genomic risk factors or new variants. Therefore, to 


\begin{abstract}
effectively control this high impact epidemic of obesity likely requires a more detailed genetic and epigenetic analysis of families with obesity and analysis of isolated populations, as well as a more thorough investigation of chemicals capable of being metabolic disruptors in this regard. Thus, the long-term solution(s) to the obesity epidemic will require a concerted multidisciplinary approach that may be more complex than just becoming more active and avoiding sedentary behavior.
\end{abstract}

\title{
1. INTRODUCTION
}

\subsection{The Current Situation}

Currently, obesity is considered an epidemic in the developed world, with the United States a country with $>30 \%$ of the population obese based on body mass index (BMI) (discussed in $[1,2]$ ), and an epidemic further impacted by the current pandemic $[3,4]$. Unfortunately, other parts of the world are rapidly catching up to these disturbing statistics. Even more disturbing is the fact that childhood obesity is also rising rapidly (discussed in [5-7]), findings that portend early development of severe health risks in young populations. Some organizations have supported the designation of obesity as a disease, but whether it is a disease or a condition that has consequences for subsequent increased risk for diseases such as type 2 diabetes, cardiovascular disease, cognitive decline, and development and progression of musculoskeletal diseases such as osteoarthritis, sarcopenia, and bone diseases, remains to be clarified.

Much research over the past few decades has focused on how this epidemic has happened. The general conclusion is that much of the epidemic is blamed on poor nutrition (reliance on diets containing too much unhealthy fat and sugar) and sedentary behavior. This interpretation is complicated by the large number $(>100)$ of genetic risk factors for obesity that have been reported [8-10], and epigenetic influences also appear to be drivers, even across generations via inheritance of risk or within generations [11, 12]. However, it is likely that many if not most of the genetic factors constitute risk, but not cause. As such, the risk may not be manifested unless other contributing variables such as diet and sedentary behavior are engaged, but it remains to be determined whether other factors in modern civilizations/societies are also contributing variables.

It should be noted that it is not just obesity in adults that is concerning, but also obesity in childhood and adolescence which is also of epidemic proportions $[6,7]$. Certainly dealing with food deprivation during growth can have long term consequences regarding height, weight, the musculoskeletal system (i.e. bone, muscles, joint tissues), and the brain. However, the effect of continual food excess on growth and maturation is likely a newer phenomenon for the general population of the Earth and Western societies, and thus, could have unintended consequences for health and function across the lifespan.

In addition, it should be noted that many individuals who lose large amounts of weight while on diets then go on to often regain the weight, even when trying to slow down the process. This lack of success has frustrated both the medical community focused on obesity and its consequences, and obese individuals, many of whom would prefer a "quick fix" from the pharma industry rather than the perhaps tougher behavior and lifestyle modification route which may not yield long term benefit. In addition, it remains to be confirmed that behavior and lifestyle are the only variables at play.

\subsection{Background}

As mentioned above, it is reported that there are $>100$ genetic risk factors for obesity (discussed in $[10,13])$. In addition, it is likely that epigenetic factors can also figure into multigenerational risk for obesity and related conditions (discussed in [14, 15]), as well as other factors (discussed in [16]). The presence of such a large number of genetic and epigenetic risk factors for obesity has led some health care providers, and likely some patients to "throw up their hands in frustration" and attempt to deal with the issues in other ways (discussed in more detail below). 
On the other hand, the large number of potential genetic and epigenetic risk factors is very telling regarding the evolutionary processes that led to what we now call Homo sapiens, or modern humans. The large number of potential risk factors is likely the accumulation of such variants which have contributed to the survival of individuals within specific subsets of populations, or accumulated in part from previous iterations of humanoids in the lineage of Homo sapiens. However, even within Homo sapiens, distinct subpopulations that have been isolated for thousands of years could have evolved unique risk signatures that were dependent on specific dietary factors and food insecurity.

It is likely that many of the genetic risk factors were related to food insecurity during evolution, and were associated with the diets involved (high protein meat, high starch plants and tubers), as well as a combination of the above after domestication of animals and development of farming. Thus, one may expect that the range of genetic risk factors may be different in different subsets of survivalist societies or cultures. Thus, after $\sim 10,000$ years those genetic risk factors in the Inuit of Canada may be different from those in survivalist cultures in Central and South America. Similarly, the profile of risk phenotype in those of European or Asian descent may also be different from those of isolated survivalist cultures. In addition to pure survival, it is also likely that some of the risk was associated with those undergoing growth and maturation, while others were associated with reproductive success in the post-puberty age from adolescence and skeletal maturity.

Given the above discussion, one could ask whether all of the genetic risk factors identified are relevant to all people with obesity, or only some of them in the modern context, and are some directly linked or indirectly linked via the consequences of obesity in the modern context? This remains to be determined.

However, there may be one important point regarding the $>100$ genetic risk factors reported that is different from why they arose, and their impact on the obesity "epidemic" of today's Western/Developed societies. That is, they may have arisen to address acute bouts of food insecurity that occur due to seasonal variation in food shortages, shortages in some foods due to over hunting or poor crop yields some years with a need for rationing, or even migrating to new locations to improve food security. Thus, having genetic/epigenetic tools to enhance food storage and retain the energy content of foods to successfully get through shortages may be a very different scenario from development of chronic obesity which may convert the multitude of genetic risk factors into the chronic form with associated acute-to-chronic modifications which make turning back either difficult or not possible! In other chronic diseases, the development of a chronic disease or state is often accompanied by epigenetic alterations that can change the landscape dramatically [17-19].

\section{POSSIBLE SOLUTIONS TO THE PROBLEMS ASSOCIATED WITH OBESITY}

Many patients consider bariatric surgery to address their obesity [20-23]. Variation in the type of bariatric surgery that is used can depend on the surgeon and the site that the surgery is performed. While the numbers of patients that qualify for such surgery appears to vary with the country, who pays, and likely in Canada, the Province of residence. In Alberta, access to bariatric surgery is somewhat limited with estimates of only 1 in 300 potential patients actually receiving the surgery after a prolonged screening period. Therefore, for patients considering this option, demand likely out paces the resources allocated in the health care system to make this a readily available option in Canada. This is also a drastic option because as a consequence it leads to significant alterations in food intake, and the physiologic dynamic and regulatory networks regarding food, the gut and the brain.

The good news is that with proper motivation, good nutrition, combined with an appropriate exercise program (tailored to the individual as not all people will respond to the same protocols; discussed in [24]), a subset of individuals can both lose weight and keep it off for an extended period of time. This can be very challenging for most individuals who have chronic obesity, and many finally give up and suffer the consequences. However, the benefits of such an integrated program can be very beneficial for mitigating insulin resistance and enhancing cardiovascular function, as well as slowing the rate of cognitive decline [25-27]. Combining diet modifications with exercise can not only enhance weight loss, but also contribute to re- 
gaining physiological fitness. In fact, some authors advocate exercise and good nutrition to inhibit the consequences of obesity even if large losses in weight are not achieved [28]. While "fit but fat" is certainly not the preferred outcome, it is likely better than not being fit!

In the case of patients with both obesity and knee osteoarthritis (OA), a diet and exercise program resulting in a $10 \%$ weight loss can lead to inhibition of OA progression and a decline in pain, with the latter also leading to lower use of pain medications. In this era of concern about opioid addiction, this latter point is an important outcome. Even for those with OA, exercise programs such as the GLA:D program [29] can provide beneficial results for improving function, lessening pain, and reducing the need for strong medications ( $\sim 35 \%$ decline in opioid medications and a more than $50 \%$ in use in general [30]). However, not everyone responds to programs such as GLA: D, so individual response patterns may dictate the need for further assessment of individual-specific exercise programs.

A key component of the success of diet and exercise programs that is often overlooked is the behavior modifications that are required to maintain the success and contribute to compliance. Thus, telling a patient with knee or hip osteoarthritis to go away and lose 100 pounds before they can be considered for surgery without access to the resources required to accomplish such a task is likely futile in many cases. For some, an acute intervention such as bariatric surgery is the only option. However, while such surgery is an option for people with insurance and available resources, it is not always an option for many individuals who may benefit from such surgery.

From the above discussion, it is clear that much effort to date has gone into the "how" to treat individuals with obesity with the goal of losing weight to prevent development or progression of disease consequences of the obesity. It is also clear that many people are in need of assistance to stem the advance of this "modern epidemic" and its impact on the health of these people and the ability of health care systems to deal with it. However, as discussed above, there are several critical questions that need to be addressed if we are to actually make inroads in this quest to stem the advance of this epidemic. The first subset of questions relates to how we can: 1) prevent it from continuing; and 2) how to prevent it from occurring in the first place.

Prevention of obesity development likely can build on and use the knowledge that appropriate exercise protocols and good nutrition can be an effective intervention scheme for a subset of those who have obesity. However, implementing prevention programs at an early age, such as in elementary schools, youth athletic programs, and via education vehicles for children and parents may be the most effective integrated approach. Implementation at an early age should and could promote healthy lifestyles across the lifespan, likely with continued reinforcement from the education system, governments, and NGOs.

Evidence in support of this perspective has come from some preclinical models. Thus, in an adult rat diet-induced obesity model (reviewed in [31]), providing a very moderate exercise protocol at the time of exposure to the high fat, high sucrose (HFS diet) completely prevents the onset of joint damage, and other sequelae of the diet on muscle changes, insulin resistance, and gut microbiome alterations [32, 33]. Interestingly, providing a prebiotic (oligofructose, inulin) which targets both the gut microbiome and also results in short chain fatty acids being released from gut microbiota which can subsequently exert a positive impact on the brain (discussed in [31]), was equally effective as exercise [32,33]. In contrast, providing the exercise protocol or the prebiotic after the diet-induced obesity has been established, was only partially effective in reversing aspects of the metabolic disturbances induced by the diet [33]. Recently, a pilot study designed to assess the effectiveness of the prebiotic on patients with both knee OA and obesity was initiated, and found to exert effectiveness in some patients [34]. Thus, while prevention is likely the best option, exercise, nutrition and prebiotics can exert significant impact after the fact.

\section{THE CRITICAL ISSUE OF WHY THE OBESITY EPIDEMIC IS HAPPENING}

A second subset of critical questions that need solving is related to asking "why" obesity has become such an epidemic, particularly over the past several decades? Without some serious thought as to the why so many people are at risk to develop obesity, and the acknowledgement that obesity is a "modern epidemic", the development of rational approaches to identifying solutions to the problem will be a daunting 
task. Certainly, having $>100$ genetic risk factors plus inheritance of epigenetic modifications leading to enhanced risk complicates finding a single solution that applies to all.

Furthermore, while obese children/adolescents and adults both appear to respond to fibre diet interventions similarly (discussed in $[34,35]$ ), it is also likely that becoming obese during maturation and onset of puberty may impart unique aspects to obesity compared to adults, but details in this regard are still unclear [5]. While some obesity-risk may be inherited via epigenetic mechanisms from either parent (discussed in $[15,36])$, whether unique epigenetic mechanisms arise with childhood obesity prior to puberty or skeletal and physiological maturity, whether there are sex-specific epigenetic changes occurring, and whether the epigenetic changes occurring as a consequence of childhood obesity differ from those arising during adult obesity, largely remain the subject of current research.

A key to perhaps better understanding regarding the WHY of the obesity epidemic is to examine the differences between the modern situation and those that likely contributed to the risks that were acquired during evolution. As discussed above, this may relate to the survival advantage of having the ability to address ACUTE needs for energy storage versus the modern day scenario of CHRONIC or inappropriate excess food intake (high fat, high sugar, prepared foods) coupled with sedentary behaviour. Therefore, in preclinical models it could provide insights if one put animals on a high fat-high sugar diet (HFHS) for various periods of time to assess whether there is a temporal transition point for the conversion from acute over nutrition to a chronic condition leading to a difficult to reverse obesity. Starting on an HFHS diet for varying periods of time and then reverting back to a more optimal diet may also allow identification of associated genetic contributors to the conversion from an acute response to a chronic obesity outcome.

An additional possibility related to the WHY of the modern obesity epidemic is that new chemicals in the modern environment (i.e. food additives, air pollution, water supplies, synthetic compounds in clothing, rugs, furniture) are contributing to the increased sensitivity to ancient obesity risk factors via disruption of metabolic control locally or via brain circuitry, making some individuals more responsive to energy imbalances and types of food leading to obesity. Certainly, hormone disruptors are known to occur in the environment [reviewed in 37], and obesity/overweight can also be a consequence of menopause [38]. An additional recent example from Crillio et al. [39] who reported that exposure to the environmental chemical DDT decades prior can lead to multi-generational higher risk for cancer and obesity also supports this concept of environmental disruptors of metabolism contributing to obesity risk. Thus, it is not beyond the realm of possibility that a combination of genetic risk and exposure to a metabolic disruptor at a specific stage of life could accelerate the development of chronic obesity, and potentially impede the non-surgical responses to address the obesity.

\section{THE PATH FORWARD AND CONCLUSIONS}

However, a major question still remaining is "why do humans have so many genetic risk factors, and what is the advantage to the species to invoke epigenetic modifications associated with risk for obesity that can be passed down to subsequent generations"? What is the purpose of so many genetic variations or risk factors versus only a few? Certainly, not all individuals have all of these risk factors, so while they are risk factors in the current context, humans are very heterogeneous in this regard, Thus, the retention of such genetic risk in the human genome likely means that such risk in today's environment was not necessarily a detrimental risk in the past, and actually imparted survival advantage to individuals, possibly due to food insecurity [40] as discussed above, and for women, an ability to carry offspring to term and supply it with milk, or other such variables that preceded the dawn of the industrial revolution with its regimented "breakfast-lunch-dinner" eating, the advent of food security, and a decline in the requirement for physical labor in the performance of many occupations. The spectrum of genetic factors that we consider obesity risk factors may have arisen from living in different locations, arisen in different populations with different diets, and may have arisen longitudinally throughout the evolutionary process over eons and accumulated as long as they served a purpose. Thus, the holdover of genetic factors and epigenetic mechanisms that contribute to risk for development of obesity in our changing environment may have contributed to our 
survival during the evolution of Homo sapiens and their predecessors have been retained in modern humans.

Therefore, the obesity epidemic may be an epidemic of our own making, and rather than medicalizing it, we should focus on improving our understanding of its fundamental basis. Better understanding of the why we still have this risk may lead to development of better strategies to mitigate these risks. In this regard, it is also apparent that not all individuals have all of the genes contributing to risk, and thus, precision or individualized health analysis may lead to individualized solutions. However, this complexity is also an opportunity to develop new understanding regarding where humans came from in order to then translate the information into more healthy individuals, and individuals who will require less reliance on health care systems.

Thus, it is likely that more detailed investigation of the genetics and epigenetics of multi-generational families with obesity could provide further clues regarding the molecular basis for obesity. In addition, such studies should also include representatives from diverse societies (i.e. Asian, African, European, Survivalist societies from around the world) who have different evolutionary histories, very different diets (i.e. starch-based, native meat, prepared food), geography (urban, rural, isolated/remote) and activity levels.

Until such long-term goals are achieved, combinations of effective exercise programs, optimized nutrition, and prebiotics to address gut microbiome issues are likely the best options for the treatment of those with early or established obesity and for prevention of obesity development in developed countries. Even if the optimized nutrition component requires some "trial and error" for specific individuals or those with specific societal backgrounds, the programs should all contain exercise programs that serve as the fundamental component of any program as exercise/mobility is central to who we are as a species [24]. However, we should not be content with that short term approach and continue to address the fundamental basis for the problems using multidisciplinary research efforts. We do not have to know every detail about a condition in order to problem solve, but in the long run, such detail will be beneficial to developing precision-focused evidence-based protocols to stem this obesity epidemic and rationally prevent its continuing. In the interim, prevention is the best strategy, and implementation of exercise programs, protection of "physical education" in schools, and nutritional education are critical for success. Thus, building on the success of school-based programs such as Action Schools! BC in British Columbia [41, 42], and others elsewhere, it should be possible to mount a concerted and effective effort to prevent obesity starting at an early age. Clearly, mitigating risk is a better approach than trying to reverse risk that has been elaborated as such expression of risk likely changes the "ground rules" for an individual due to the impact of obesity on multiple physiologic systems.

Finally, in the future it may be possible to reverse some epigenetically modified states using targeted drug approaches. Thus, being able to eliminate or reverse many such changes associated with the chronic state of obesity could make the approaches outlined above more effective for those with established obesity, improve their quality of life, and diminish their risk for other co-morbidities later in life.

\section{ACKNOWLEDGEMENTS}

The author thanks many colleagues for interesting discussions on the topic, as well as the participants of a 2017 workshop on Obesity and Osteoarthritis supported by the Alberta Health Services (AHS) Bone \& Joint Health Strategic Clinical Network. The author also thanks the AHS Strategic Clinical Network Program for salary and grant support, as well as grant support from the University of Calgary McCaig Institute for Bone \& Joint Health.

\section{CONFLICTS OF INTEREST}

The author declares that he has no conflicts of interest to disclose.

\section{REFERENCES}

1. Fulton, M. and Srinivan, V.N. (2021) Obesity, Stigma and Discrimination. StatPearls [Internet], March 16. 
2. Santos, A.L. and Sinha, S. (2021) Obesity and Aging: Molecular Mechanisms and Therapeutic Approaches. Ageing Research Reviews, 67, Article ID: 101268. https://doi.org/10.1016/j.arr.2021.101268

3. Cefalu, W.T. and Rodgers, G.P. (2021) COVID-19 and Metabolic Diseases: A Heightened Awareness of Health Inequities and Renewed Focus for Research Priorities. Cell Metabolism, 33, 473-478. https://doi.org/10.1016/j.cmet.2021.02.006

4. Sumbria, D., Berber, E., Mathayan, M. and Rouse, B.T. (2021) Virus Infections and Host Metabolism-Can We Manage the Interactions? Frontiers in Immunology, 11, Article ID: 594963. https://doi.org/10.3389/fimmu.2020.594963

5. Collins, K.H., MacDonald, G.Z., Hart, D.A., Seerattan, R.A., Rios, J.L., Reimer, R.A. and Herzog, W. (2020) Impact of Age on Host Responses to Diet-Induced Obesity: Development of Joint Damage and Metabolic Set Points. Journal of Sport and Health Science, 9, 132-139. https://doi.org/10.1016/j.jshs.2019.06.004

6. Verduci, E., Bronsky, J., Embleton, N., Gerasimidis, K., Indrio, F., Koglmeier, J., et al. (2021) Role of Dietary Factors, Food Habits and Lifestyle in Childhood Obesity Development. A Position Paper from the European Society for Paediatric Gastroenterology, Hepatology and Nutrition Committee on Nutrition. Journal of Pediatric Gastroenterology and Nutrition. Online Ahead of Print. https://doi.org/10.1097/MPG.0000000000003075

7. Steinhart, A., Tsao, D. and Pratt, J.S.A. (2021) Pediatric Metabolic and Bariatric Surgery. Surgical Clinics of North America, 101, 199-212. https://doi.org/10.1016/j.suc.2020.12.007

8. Van Dijk, S.J., Molloy, P.L., Varinli, H., Morrison, J.L. and Muhlhausler, B.S. (2015) Epigenetics and Human Obesity. International Journal of Obesity, 39, 85-97. https://doi.org/10.1038/ijo.2014.34

9. Qasim, A., Turcotte, M., de Souza, R.J., Samaan, M.C., Champredon, D., Dushoff, J., et al. (2017) On the Origin of Obesity: Identifying the Biological, Environmental and Cultural Drivers of Genetic Risk among Human Populations. Obesity Reviews, 19, 121-149. https://doi.org/10.1111/obr.12625

10. Thaker, V.V. (2017) Genetic and Epigenetic Causes of Obesity. Adolescent Medicine. State of the Art Reviews, 28, 379-405.

11. Ahmed, F. (2010) Epigenetics: Tales of Adversity. Nature, 468, S20. https://doi.org/10.1038/468S20a

12. Saban, K.L., Mathews, H.L., DeVon, H.A. and Janusek, L.W. (2014) Epigenetics and Social Context: Implications for Disparity in Cardiovascular Disease. Aging and Disease, 5, 346-355. https://doi.org/10.14336/AD.2014.0500346

13. Froguel, P. (2015) Obesity Genes and Their Effect on the Energy Balance. Bulletin de L'Académie Nationale de Médecine, 199, 1269-1279. (In French) https://doi.org/10.1016/S0001-4079(19)30812-X

14. Paul, H.A., Collins, K.H., Nicolucci, A.C., Urbanski, S.J., Hart, D.A., Vogel, H.J. and Reimer, R.A. (2019) Maternal Prebiotic Supplementation Reduces Fatty Liver Development in Offspring through Altered Microbial and Metabolic Profiles in Rats. The FASEB Journal, 33, 5153-5167. https://doi.org/10.1096/fj.201801551R

15. Ryan, C.P. and Kusawa, C.W. (2020) Germline Epigenetic Inheritance: Challenges and Opportunities for Linking Human Paternal Experience with Offspring Biology and Health. Evolutionary Anthropology, 29, 180-200. https://doi.org/10.1002/evan.21828

16. Sauder, K.A. and Ritchie, N.D. (2021) Reducing Intergenerational Obesity and Diabetes Risk. Diabetologia, 64, 481-490. https://doi.org/10.1007/s00125-020-05341-y

17. Aloia, L. (2021) Epigenetic Regulation of Cell-Fate Changes That Determine Adult Liver Regeneration after Injury. Frontiers in Cell and Developmental Biology, 9, Article ID: 643055. https://doi.org/10.3389/fcell.2021.643055

18. Tam, A.K.M. and Leung, D.C.Y. (2021) Epigenomic and Transcriptomic Analysis of Chronic Inflammatory Disease. Genes Genomics, 43, 227-236. https://doi.org/10.1007/s13258-021-01045-8 
19. Pereira, B.M.V., Katakia, Y.T., Majumder, S. and Thieme, K. (2021) Unraveling the Epigenetic Landscape of Glomerular Cells in Kidney Disease. Journal of Molecular Medicine (Berlin, Germany). Online Ahead of Print. https://doi.org/10.1007/s00109-021-02066-2

20. Chalklin, C.G., Ryan-Harper, E.G. and Beamish, A.J. (2021) Metabolic and Bariatric Surgery in Adolescents. Current Obesity Reports. Online Ahead of Print. https://doi.org/10.1007/s13679-021-00423-3

21. Roberts, C.A. (2021) Physical and Physcological Effects of Bariatric Surgery on Obese Adolescents: A Review. Frontiers in Pediatrics, 8, Article ID: 591598. https://doi.org/10.3389/fped.2020.591598

22. Bhandari, M., Kosta, S., Khurana, M., Mathur, W., Reddy, M.K. and Fobi, M. (2021) Emerging Procedures in Bariatric Metabolic Surgery. Surgical Clinics of North America, 101, 335-353. https://doi.org/10.1016/j.suc.2020.12.001

23. Kim, Y.J., Womble, J.T., Gunsch, C.K. and Ingram, J.L. (2021) The Gut/Lung Microbiome Axis in Obesity, Asthma, and Bariatric Surgery: A Literature Review. Obesity (Silver Springs), 29, 636-644. https://doi.org/10.1002/oby.23107

24. Hart, D.A. and Zernicke, R.F. (2020) Optimal Human Functioning Requires Exercise across the Lifespan: Mobility in a 1g Environment Is Intrinsic to the Integrity of Multiple Biologic Systems. Frontiers in Physiology, 11, 156. https://doi.org/10.3389/fphys.2020.00156

25. Barha, C.R. and Liu-Ambrose, T. (2020) Sex Differences in Exercise Efficacy: Is Midlife a Critical Window for Promoting Healthy Cognitive Aging? The FASEB Journal, 34, 11329-11336. https://doi.org/10.1096/fj.202000857R

26. Barha, C.R., Falck, R.S., Skou, S.T. and Liu-Ambrose, T. (2021) Personalizing Exercise Recommendations for Healthy Cognition and Mobility in Aging: Time to Address Sex and Gender (Part 1). British Journal of Sports Medicine, 55, 300-301. https://doi.org/10.1136/bjsports-2020-102864

27. Barha, C.R., Falck, R.S., Shou, S.T. and Liu-Ambrose, T. (2021) Personalizing Exercise Recommendations for Healthy Cognition and Mobility in Aging: Time to Consider One's Pre-Existing Function and Genotype (Part 2). British Journal of Sports Medicine, 55, 301-303. https://doi.org/10.1136/bjsports-2020-102865

28. Glaesser, G.A. and Blair, S.N. (2018) The Health Risks of Obesity Have Been Exaggerated. Medicine \& Science in Sports \& Exercise, 51, 218-221. https://doi.org/10.1249/MSS.0000000000001746

29. Roos, E.M., Gronne, D.T., Skou, S.T., Zywiel, M.G., McGlasson, R., Barton, C.J., Kemp, J.L., Crossley, K.M. and Davis, A.M. (2021) Immediate Outcomes Following the GLA:D Program in Denmark, Canada and Australia. A Longitudinal Analysis Including 28370 Patients with Symptomatic Knee or Hip Osteoarthritis. Osteoarthritis Cartilage. https://doi.org/10.1016/j.joca.2020.12.024

30. Thorlund, J.B., Roos, E.M., Goro, P., Gromelsky Ljungcrantz, E., Gronne, D.T. and Skou, S.T. (2020) Patients Use Fewer Analgesics Following Supervised Exercise Therapy and Patient Education: An Observational Study of 16499 Patients with Knee or Hip Osteoarthritis. British Journal of Sports Medicine. Online Ahead of Print. https://doi.org/10.1136/bjsports-2019-101265

31. Collins, K.H., Herzog, W., MacDonald, G.Z., Reimer, K.A., Rios, J.L., Smith, I.C., Zernicke, R.F. and Hart, D.A. (2018) Obesity, Metabolic Syndrome, and Musculoskeletal Disease: Common Inflammatory Pathways Suggest a Central Role for Loss of Muscle Integrity. Frontiers in Physiology, 239, 112. https://doi.org/10.3389/fphys.2018.00112

32. Rios, J.L., Bomhof, M.R., Reimer, R.A., Hart, D.A., Collins, K.H. and Herzog, W. (2019) Protective Effect of Prebiotic and Exercise Intervention on Knee Health in a Rat Model of Diet-Induced Obesity. Scientific Reports, 9, Article No. 3893. https://doi.org/10.1038/s41598-019-40601-x

33. Rios, J.L., Hart, D.A., Reimer, R.A. and Herzog, W. (2020) Prebiotic and Exercise Do Not Alter Knee Osteoarthritis in a Rat Model of Established Obesity. Cartilage. Online Ahead of Print. 


\section{https://doi.org/10.1177/1947603520959399}

34. Fortuna, R., Hart, D.A., Sharkey, K.A., Schachar, R.A., Johnston, K. and Reimer, R.A. (2021) Effect of a Prebiotic Supplement on Knee Joint Function, Gut Microbiota and Inflammation in Adults with Co-Morbid Obesity and Knee Osteoarthritis: Study Protocol for a Randomized Controlled Trial. Trials. In Press. https://doi.org/10.1186/s13063-021-05212-w

35. Nicolucci, A.C., Hume, M.P., Martinez, I., Mayengbam, S., Walter, J. and Reimer, R.A. (2017) Prebiotics Reduce Body Fat and Alter Intestinal Microbiota in Children Who Are Overweight or with Obesity. Gastroenterology, 153, 711-722. https://doi.org/10.1053/j.gastro.2017.05.055

36. Jawaid, A., Jehle, K.L. and Mansuy, I.M. (2021) Impact of Parental Exposure on Offspring Health in Humans. Trends in Genetics, 37, 373-388. https://doi.org/10.1016/j.tig.2020.10.006

37. Kiess, W., Haussler, G. and Vogel, M. (2021) Endocrine-Disrupting Chemicals and Child Health. Best Practice \& Research Clinical Endocrinology \& Metabolism. Online Ahead of Print. https://doi.org/10.1016/j.beem.2021.101516

38. Ko, S.H. and Kim, H.S. (2020) Menopausal-Associated Lipid Metabolic Disorders and Foods Beneficial for Postmenopausal Women. Nutrients, 12, 202. https://doi.org/10.3390/nu12010202

39. Crillio, P.M., La Merrill, M.A., Krigbaum, N.Y. and Cohn, B.A. (2021) Grandparental Perinatal Serum DDT in Relation to Granddaughter Early Menarche and Adult Obesity: Three Generations in the Child Health and Development Studies Cohort. Cancer Epidemiology, Biomarkers \& Prevention. Online Ahead of Print. https://doi.org/10.1158/1055-9965.EPI-20-1456

40. Nettle, D., Andrews, C. and Bateson, M. (2017) Food Insecurity as a Drive of Obesity in Humans: The Insurance Hypothesis. Behavioral and Brain Sciences, 40, e105. https://doi.org/10.1017/S0140525X16000947

41. Naylor, P.J. and McKay, H.A. (2009) Prevention in the First Place: Schools a Setting for Action on Physical Activity. British Journal of Sports Medicine, 43, 10-13. https://doi.org/10.1136/bjsm.2008.053447

42. McKay, H.A., MacDonald, H.M., Nettleford, L., Masse, L.C., Day, M. and Naylor, P.J. (2015) Action Schools! BC. British Journal of Sports Medicine, 49, 210-218. https://doi.org/10.1136/bjsports-2013-093361 